Bundesgesundheitsbl 2016 · 59:1660-1661

DOI 10.1007/s00103-016-2464-y

C) Springer-Verlag Berlin Heidelberg 2016

Bekanntmachung des Umweltbundesamtes

\title{
Stellungnahme der Innenraumhygienekommission (IRK) zu elektronischen Zigaretten (E-Zigaretten)
}

Verdampfungsprozess wird durch Tastendruck und durch Ansaugen am Mundstück aktiviert. Die E-Zigarette imitiert in ihrer Handhabung und zumeist auch in ihrer Optik die Tabakzigarette. Freisetzungsprozesse wie bei der glimmenden Tabakzigarette über den Nebenstromrauch sind bei der elektronischen Zigarette zu vernachlässigen. Das Aerosol wird nur dann gebildet, wenn der Konsument die E-Zigarette durch Saugen oder Tastendruck aktiviert. Die Substanzen aus dem Liquid gelangen daher nur über das Exhalat (die ausgeatmete Luft) des Konsumenten in die Raumluft.

Die Innenraumhygienekommission des Umweltbundesamtes hat die Frage diskutiert, ob es analog zum Passivrauchen („passive smoking“) auch ein „Passivdampfen“ („passive vaping“) gibt, und ob exhalierte E-Zigarettendämpfe die Raumluft verunreinigen und die Gesundheit von Dritten gefährden können.

Beim E-Zigarettenkonsum sorgt die Trägersubstanz Propylenglykol für den sichtbaren Dampf beim Ausatmen. Aus dem in der Atemluft übersättigten Propylenglykol bilden sich feine und ultrafeine Flüssigkeitspartikel (Aerosole), deren Größenverteilung und Anzahlkonzentrationen denen des Tabakrauchs ähneln [6]. Nach aktuellen Untersuchungsergebnissen des Bayerischen Landesamtes für Gesundheit und Lebensmittelsicherheit steigen beim Gebrauch von E-Zigaretten die Raumluftkonzentrationen von feinen $\left(\mathrm{PM}_{2,5}\right)$ und ultrafeinen Partikeln (UFP) an [8]. Die ultrafeinen Partikel dringen bis in die Alveolen der Lunge ein [7]. Darüber hinaus lag nach zweistündigem E-Zigaretten-Gebrauch Propylenglykol in der
Raumluft in einer Konzentration vor, die den vorläufigen Richtwert I (RW I) von $0,07 \mathrm{mg} / \mathrm{m}^{3}$ [16] um etwa das Dreifache überschritt [8]. Aus Gründen der Vorsorge sollte dies vermieden werden.

In einem Raum mit intensivem E-Zigarettenkonsum kann die Belastung der Raumluft mit $\mathrm{PM}_{2,5}$ auf Werte ansteigen, wie sie in Gaststätten mit erlaubtem $\mathrm{Zi}$ garettenkonsum gemessen werden bzw. wurden [9]. Aus epidemiologischen Studien ist bekannt, dass $\mathrm{PM}_{2,5}$ Atemwegs- und Herz-Kreislauferkrankungen verursacht $[10,11]$. Besonders empfindlich reagieren Personen mit bestehender Herz- oder Lungenerkrankung, ältere Menschen und Kinder. Bei Kindern beeinträchtigt eine Belastung mit $\mathrm{PM}_{2,5}$ die Lungenentwicklung. Dabei sind bisher keine Konzentrationen bekannt, unterhalb derer keine Gesundheitsschäden auftreten. Obwohl sich die Partikel im E-Zigarettenaerosol in ihrer Beschaffenheit von denen aus der Umwelt und auch denen des Tabakrauchs unterscheiden, ist davon auszugehen, dass sie aufgrund ihrer chemischen Zusammensetzung und Morphologie die Gesundheit beeinträchtigen können [12]. In der Zellkultur und im Tierversuch konnte jüngst nachgewiesen werden, dass Partikel im Aerosol von E-Zigaretten oxidativen Stress und Entzündungsreaktionen hervorrufen [13]. Entsprechende Untersuchungen am Menschen fehlen bislang.

Propylenglykol ist zwar als Lebensmittelzusatzstoff zugelassen und für den oralen Gebrauch (Aufnahme über den Mund) unbedenklich, zur Auswirkung einer inhalativen Aufnahme gibt es jedoch keine Langzeitstudien am Menschen. Angestellte in der Unterhaltungsindustrie, 
die regelmäßig propylenglykolhaltigen Aerosolen wie Theaternebel ausgesetzt sind, leiden aber häufiger an Atemwegsreizungen und Einschränkungen der Lungenfunktion als nicht exponierte Personen [14]. Vernebeltes Propylenglykol löst bereits bei kurzfristiger Exposition Augen- und Atemwegsirritationen aus [15].

Im Aerosol der meisten Liquids finden sich auch krebserzeugende Substanzen wie Formaldehyd, Benzol und tabakspezifische Nitrosamine sowie möglicherweise krebserzeugende Substanzen wie Acrolein und Acetaldehyd. Die meisten dieser Substanzen entstehen erst durch die Verdampfung der Liquids. Die Komponenten lassen sich in der Raumluft deutlich nachweisen, die Einzelkonzentrationen sind dennoch gering [8].

Mehrere Untersuchungen wiesen $\mathrm{Ni}$ kotin in der Raumluft nach, wenn nikotinhaltige E-Zigaretten konsumiert wurden [12]. Laut einer Untersuchung des Bayerischen Landesamtes für Gesundheit und Lebensmittelsicherheit führt Nikotin im Aerosol bereits nach nur fünfminütigem E-Zigarettengebrauch bei den Konsumenten zu einer Erhöhung von Stickstoffmonoxid (NO) in der Atemluft [8]. $\mathrm{NO}$ entsteht während Entzündungsprozessen der Epithelzellen und gilt als effektiver Anzeiger einer Atemwegsentzündung. Eine Vielzahl von Tier- und Zellkulturversuchen weist zudem darauf hin, dass Nikotinkonsum - neben der gesicherten suchterzeugenden Wirkung - langfristig Gesundheitsschäden hervorruft [17, 18]. Passiver Nikotinkonsum des Fötus im Mutterleib schadet dem Ungeborenen und wird mit dem Plötzlichen Kindstod [19] und Entwicklungsstörungen des heranwachsenden Gehirns in Verbindung gebracht [20]. Aus diesem Grund gelten in Deutschland sehr strenge Anforderungen an den Gehalt und den Konsum von nikotinhaltigen Erzeugnissen. E-Zigaretten sind hier nach Auffassung der Innenraumlufthygiene-Kommission in keiner Weise weniger schädlich als herkömmliche Zigaretten.

Unter Auswertung aller Aspekte und auf Basis der aktuellen Studienlage kommt die IRK zu der Einschätzung, dass nikotinhaltige und nikotinfreie EZigaretten zur Belastung der Raumluft mit gasförmigen organischen Verbin- dungen und Partikeln beitragen. Nach Einschätzung der IRK kann das „Passivdampfen“ die Gesundheit von Dritten gefährden. Auch wenn das genaue Ausmaß der Gefährdung bislang nicht eindeutig geklärt ist, empfiehlt die IRK, dass Bestimmungen und Beschränkungen, die für herkömmliches Tabakrauchen gelten, auch für E-Zigaretten anzuwenden und diese in das Bundesnichtraucherschutzgesetz und in die Nichtraucherschutzgesetze der Länder aufzunehmen.

\section{Literatur:}

1. Schaller K, Braun S, Kahnert S, Viarisio V, PötschkeLanger M (2015) Use of electronic cigarettes in Germany. Poster, 19.03.2015, 16th World conference on Tobacco or Health, Abu Dhabi, Vereinigte Arabische Emirate

2. Bundeszentrale für gesundheitliche Aufklärung (2015) Verbreitung des Rauchens von E-Shishas, E-Zigaretten und Tabak-Zigaretten bei 12- bis 17-Jährigen Jugendlichen in Deutschland. Sonderauswertung der Repräsentativbefragung "Alkoholsurvey 2014"

3. Bundesregierung (2016) Besserer Schutz vor E-Shishas. https://www.bundesregierung.de/Content/DE/Artikel/2015/11/2015-11-04-e-zigarettenshishas.html. Zugegriffen: 26.Okt.2016

4. Deutscher Bundestag (2012) Antwort der Bundesregierung auf die Kleine Anfrage der Abgeordneten Dr. Martina Bunge, Diana Golze, Karin Binder, weiterer Abgeordneter und der Fraktion DIE LINKE. Drucksache 17/8652. Gesundheitliche und rechtliche Bewertung von E-Zigaretten

5. Schripp T, Markewitz D, Uhde E, Salthammer T (2013) Does e-cigarette consumption cause passive vaping? Indoor Air 23:25-31

6. Fuoco FC, Buonanno G, Stabile L, Vigo P (2014) Influential parameters on particle concentration and size distribution in the mainstream of e-cigarettes. Environ Pollut 184:523-529

7. Manigrasso M, Buonanno G, Fuoco FC, Stabile L, Avino P (2014) Aerosol deposition doses in the human respiratory tree of electronic cigarette smokers. Environ Pollut 196(C):257-267

8. Schober W, Szendrei K, Matzen W, Osiander-Fuchs $H$, Heitmann D, Schettgen T, Jörres RA, Fromme H (2014) Use of electronic cigarettes (e-cigarettes) impairs indoor air quality and increases FeNO levels of e-cigarette consumers. Int J Hyg Environ Health 217:628-637

9. Soule EK, Maloney SF, Spindle TR, Rudy AK, Hiler MM, Cobb CO (2016) Electronic cigarette use and indoor air quality in a natural setting. Tob Control. Tob Control. doi:10.1136/tobaccocontrol-2015-052772

10. Stanek LW, Sacks JD, Dutton SJ, Dubois JJB (2011) Attributing health effects to apportioned components and sources of particulate matter: an evaluation of collective results. Atmos Environ 45:5655-5663

11. WHO Regional Office for Europe, Copenhagen (2013) Health effects of particulate matter. World Health Organization. http://www.euro.who.int/_
data/assets/pdf_file/0006/189051/Health-effectsof-particulate-matter-final-Eng.pdf. Zugegriffen: 2. März 2015

12. Deutsches Krebsforschungszentrum (Hrsg) (2015) Belastung der Innenraumluft durch Emissionen von E-Zigaretten. Aus der Wissenschaft für die Politik. Deutsches Krebsforschungszentrum, Heidelberg

13. Lerner CA, Sundar IK, Yao H, Gerloff J, Ossip DJ, McIntosh S, Robinson R, Rahman I (2015) Vapor produced by electronic cigarettes and e-juices with flavorings induce toxicity, oxidative stress, and inflammatory response in lung epithelial cells and in mouse lung. PLOS ONE 10:e0116732

14. Varughese $S$, Teschke $K$, Brauer $M$, Chow $Y$, van Netten C, Kennedy SM (2005) Effects of theatrical smokes and fogs on respiratory health in the entertainment industry. Am J Ind Med 47:411-418

15. Wieslander G, Norbäck D, Lindgren T (2001) Experimental exposure to propylene glycol mist in aviation emergency training: acute ocular and respiratory effects. Occup Environ Med 58:649-655

16. Umweltbundesamt, Ausschuss für Innenraumrichtwerte (AIR) (2013) Ergebnisprotokoll der 48. Sitzung am 04. und 05.11.2013. TOP 7: Richtwerte für Propylenglykol in der Innenraumluft. https:// www.umweltbundesamt.de/sites/default/files/ medien/378/dokumente/48_kurzprotokoll_ k1.pdf. Zugegriffen: 21. Okt. 2016

17. U.S. Department of Health and Human Services (2014) The health consequences of smoking - 50 years of progress. A report of the Sugeon General. Centers for Disease Control and Prevention, Georgia

18. Grando SA (2014) Connections of nicotine to cancer. Nat Rev Cancer 14:419-429

19. Dwyer JB, McQuown SC, Leslie FM (2009) The dynamic effects of nicotine on the developing brain. Pharmacol Ther 122:125-139

20. England LJ, Bunnell RE, Pechacek TF, Tong VT, McAfee TA (2015) Nicotine and the developing human: a neglected element in the electronic cigarette debate. Am J Prev Med 49:286-293 